\title{
Post-systolic shortening is superior to global longitudinal strain in predicting adverse events in patients with stable coronary artery disease and preserved systolic function
}

Shirui Lu, Xin Hu, Jun Zhang, Ying Zhu, Wei Zhou, Yani Liu* and Youbin Deng

\begin{abstract}
Background: Post-systolic shortening (PSS) is an important indicator for early identifying myocardial dysfunction. We aimed to investigate the predictive value of PSS assessed with speckle tracking automated functional imaging (AFI) on adverse events in patients with stable coronary artery disease (CAD) and preserved systolic function.

Methods: A total of 204 consecutive patients clinically diagnosed with stable CAD and left ventricular ejection fraction (LVEF) $>50 \%$ were included. Multiple parameters were analyzed with AFI technique. The composite endpoint included all-cause mortality, heart failure, myocardial infarction and stroke.

Results: During a median follow-up of 24 months (IQR 19-28 months), 30 patients (14.7\%) reached the endpoint. Patients experiencing the endpoint had a lower absolute global longitudinal strain (GLS), a higher post-systolic index (PSI), and more left ventricle walls displaying PSS than patients without events. PSI (hazard ratio, 1.15; 95\% Cl, 1.04$1.27 ; p=0.005$ ) and per 1 increase in the number of left ventricle walls with PSS (hazard ratio $1.52,95 \% \mathrm{Cl} 1.21-1.91$, $p<0.000$ ) were independent predictors of the endpoint, whereas GLS was not significantly associated with the endpoint after adjustment models. For patients with absolute value of $G L S>15.4 \%$, a significant prognostic superiority was found in PSI compared with GLS (AUC $=0.73$ [PSI] vs. $0.58[\mathrm{GLS}], p=0.024)$.
\end{abstract}

Conclusions: PSS is an independent predictor for adverse events in stable CAD patients with preserved systolic function, and the prognostic value may be superior to GLS in patients with normal or mildly reduced GLS.

Keywords: Stable coronary artery disease, Speckle tracking echocardiography, Automated functional imaging, Postsystolic shortening, Global longitudinal strain

\section{Key points}

- GLS and PSS can early identify myocardial dysfunction.

- PSI and LV walls with PSS can independently predict adverse outcomes.

*Correspondence: yani.liu@163.com; yani.liu@tjh.tjmu.edu.cn Department of Medical Ultrasound, Tongji Hospital, Tongji Medical College, Huazhong University of Science and Technology, 1095 Jiefang Road, Wuhan 430030, China
- PSS may have a superiority predicting adverse outcomes when GLS was preserved.

\section{Introduction}

Coronary artery disease (CAD) remains the leading cause of death in developed and developing countries [1]. Stable CAD refers to known CAD with no recent or acute events, encompassing a diverse spectrum of patients. Notably, the concept of 'stable' CAD is misleading due to the continuing risks of cardiovascular events over the 
longer term and the heterogeneous risk characteristics [2]. It has been reported that the probability of having major adverse cardiovascular events (MACEs) within five years of the onset of apparently stable angina is up to $35 \%$, depending on clinical variables that affect the risk [3]. Therefore, it is of great value to improve the abilities during the work-up in identifying those patients with increased risk of adverse outcomes.

Transthoracic echocardiography is the commonly used non-invasive imaging method for the evaluation of CAD, which was shown not only to aid diagnosis but also to provide valuable prognostic information in the clinical setting [4]. Compared to the traditional left ventricular ejection fraction (LVEF), a growing literature found that speckle tracking echocardiography (STE) could provide more sensitive parameters in early identifying subtle myocardial damage, particularly contributing to improving the prognostic assessment in the patients with preserved LVEF [5]. In a study of 659 patients after acute myocardial infarction, global longitudinal strain (GLS) was independently related to all-cause mortality, reinfarction, revascularization, and heart failure hospitalization at 3-year follow-up and was found to be superior to LVEF and wall motion score index (WMSI) [6].

In addition to longitudinal strain, post-systolic shortening (PSS) has recently attracted attention as another marker of myocardial ischemia [7]. PSS is defined as a delayed systolic shortening occurring after the aortic valve closure. The most commonly used parameter to quantify PSS is the post-systolic index (PSI) which is calculated as [8]:

PSI $=\frac{\text { maximal strain }- \text { peak systolic strain }}{\text { maximal strain }} \times 100$

where maximal strain and peak systolic strain indicate the peak negative strain in cardiac cycle and in systole, respectively.

It is reported that PSS may occur in passive dyskinesia and also at a low magnitude in healthy individuals [9]. According to Voigt et al. [10], pathological PSS is defined as at least one segment of left ventricle (LV) myocardium PSI (Eq. 1) $>20 \%$. The superiority of PSS to conventional parameters in detecting acute ischemia and predicting adverse cardiovascular events has been indicated in clinical studies $[11,12]$. Interestingly, among the myocardial deformation parameters derived from STE, PSS is sometimes observed without a relative strain decrease in the acute ischemic region, especially after recovery from ischemia [13]. In a recent animal study, PSS remained in the risk area at $20 \mathrm{~min}$ after reperfusion significantly, although peak systolic strain recovered by $20 \mathrm{~min}$ [14]. Currently, some authors consider the assessment of PSS during STE analysis in patients with CAD as equally or more critical to commonly used systolic strain, particularly in a state of acute ischemia or systolic stunning [7, 15]. However, there is still a lack of data concerning PSS and its prognostic significance compared with LVEF and systolic strain in stable CAD population.

Different from the traditional manually tracing the myocardium, a novel algorithm of STE, named automatic functional imaging (AFI), can automatically and quickly provide multiple strain parameters, in turn improving workflow and reducing user variability [16]. Therefore, AFI algorithm was used in the present study to explore the prognostic value of AFI multiple-parameter analysis in stable CAD patients with preserved ejection fraction, which would be more challenging in clinical setting. Moreover, whether PSS assessed by AFI in stable CAD patients could provide powerful prognostic information on the adverse cardiovascular events and its superiority to GLS was fully explored.

\section{Methods}

\section{Study population}

We prospectively studied 297 consecutive patients with clinically diagnosed stable CAD from April 2016 to December 2020. Inclusion criteria were (1) suspected CAD patients had stable intermittent chest pain or exertional dyspnea related to myocardial ischemia according to a comprehensive clinical investigation including location, character, duration and relationship to exertion and other exacerbating or relieving factors. (2) No changes in frequency, duration, precipitating causes or relief for at least two months before admission. All patients underwent transthoracic echocardiography combined with speckle tracking AFI analysis. Critical exclusion criteria were (1) coronary angiography revealed $<50 \%$ reduction of the arterial lumen area in any major coronary artery; (2) $\mathrm{LVEF} \leq 50 \%$; (3) a history of significant heart failure, acute coronary syndrome within the last year; (4) other heart diseases including cardiomyopathy and significant valvular heart disease, ventricular arrhythmia, atrial fibrillation, left or right bundle branch block or paced rhythm, and other serious non-cardiac diseases; (5) suboptimal image quality and arrhythmia that may influence the analysis of speckle tracking AFI. A total of 204 patients were finally included in this study. Tongji Hospital Ethics Committee approved the study, and informed consent was obtained from all patients before the study.

\section{Transthoracic echocardiography}

Transthoracic echocardiograms were performed using GE Vivid E95 ultrasound equipment (GE Vingmed Ultrasound, Horten, Norway) with an M5Sc transducer $(1.7-3.3 \mathrm{MHz})$ and a high frame rate (70-80 frame/s). LV parasternal long-axis view, short-axis views at basal, 
midventricular and apical levels, as well as apical 2-, 3 - and 4-chamber views were acquired. All echocardiograms were analyzed in accordance with the quantitative method recommended by the American Society of Echocardiography [17]. The interventricular septum (IVS) thickness, left ventricular posterior wall (LVPW) thickness and left ventricular end-diastolic dimension (LVEDD) were measured in the parasternal long-axis view at the level of mitral valve leaflet tips. LV mass index (LVMI) was calculated as the anatomic mass divided by the body surface area. Biplane Simpson's method was used to determine LV end-systolic volumes (LVESV), LV end-diastolic volumes (LVEDV) and LVEF. In the four-chamber apical view, pulsed-wave Doppler was used to assess mitral valve inflow velocities, including peak velocity of early (E) and late (A) diastolic LV filling. E/A ratio was calculated. Pulsed-wave tissue Doppler was also used to measure peak early diastolic $\left(\mathrm{e}^{\prime}\right)$ velocities at the septal mitral annular in the fourchamber view. The $\mathrm{E} / \mathrm{e}^{\prime}$ was calculated. All echocardiograms were analyzed by an investigator who was blinded to all clinical baseline and outcome data.

\section{Speckle tracking automated functional imaging}

The commercial speckle tracking AFI software (version 2.3, GE Vingmed Ultrasound) was used to perform twodimensional (2D) strain analysis in apical two-, threeand four-chamber views. The AFI software analyses myocardial motion by tracking frame-to-frame speckle changes in 2D images [18]. When necessary, automatic endocardial recognition was manually adjusted to ensure correct 'anchorage' of the algorithm to the mitral annulus, exclude papillary muscles and chordae from tracking and correctly include the LV apex. The region of interest (ROI) was eventually adjusted to ensure tracking of the whole myocardial thickness [19]. LV outflow pulsed Doppler was used to time end systole [20]. The segmental strain curves in apical view and 18-segment bull's-eye diagrams related to strain parameters were automatically displayed (Fig. 1).

GLS was calculated as the average value of the peak systolic strain in LV 18 myocardial segments [21]. PSI of each segment was calculated as Eq. (1) (Additional file 1: Fig. S1). When post-systolic shortening was not observed, PSI was set to zero. Overall PSI was obtained as the average from the 18 myocardial segments. In the categorical analysis, the presence of PSS was defined as PSI $>20 \%$ in at least one myocardial segment. This cutoff value was based on previous evidence evaluating PSS [10]. If a segment within a myocardial wall displayed PSS, the wall was categorized as having the presence of PSS.

\section{Coronary angiography}

All patients underwent coronary angiography to confirm the diagnosis with CAD. Coronary angiography was performed by two experienced interventionists using standard technique [22]. Stenosis with $\geq 50 \%$ reduction of the arterial lumen area in at least one major coronary artery (including left main artery [LM], the left anterior descending artery [LAD], left circumflex artery [LCX] and right coronary artery $[\mathrm{RCA}]$ ) was considered CAD.

\section{Follow-up}

All participants were followed from July 2016 to August 2021 or the time of the event. The composite endpoint included all-cause mortality, heart failure, myocardial infarction and stroke. If a patient experienced several events, the first occurring event was used in the composite end point. The end points were obtained from our hospital's Patient Registry and telephone calls. Fifteen patients lost to follow-up were excluded in the final analysis.

\section{Reproducibility}

To define intra- and inter-observer variability, 30 patients were randomly selected and remeasured by two observers blinded to patient data and each other's results. One of the researchers did the analysis once again after two weeks. Intra- and inter-observer reproducibility and variability were calculated by intraclass correlation coefficient (ICC).

\section{Statistical analysis}

All analyses were performed using SPSS version 21.0 statistical analysis software (IBM, Armonk, NY, USA), MedCalc version 15.2.2 (MedCalc Software, Mariakerke, Belgium) and R version 4.1.1 (R Foundation for Statistical Computing, Vienna, Austria). Normally distributed continuous data are presented as mean \pm standard deviation. Non-normally distributed continuous data are presented as median and interquartile range (IQR). Shapiro-Wilk test and q-q plots were used to check continuous values for normality of the data distribution. The differences between two groups were assessed using Student's t test for normally distributed data and Mann- Whitney $U$ test for non-normally distributed data. The differences among the three groups were analyzed using the one-way analysis of variation (ANOVA) test for normally distributed data with the Bonferroni correction for pairwise comparisons between the data in each group. The Kruskal-Wallis rank-sum test was used for non-normally distributed data in three groups, and the all pairwise method was used for further pairwise comparisons between two 

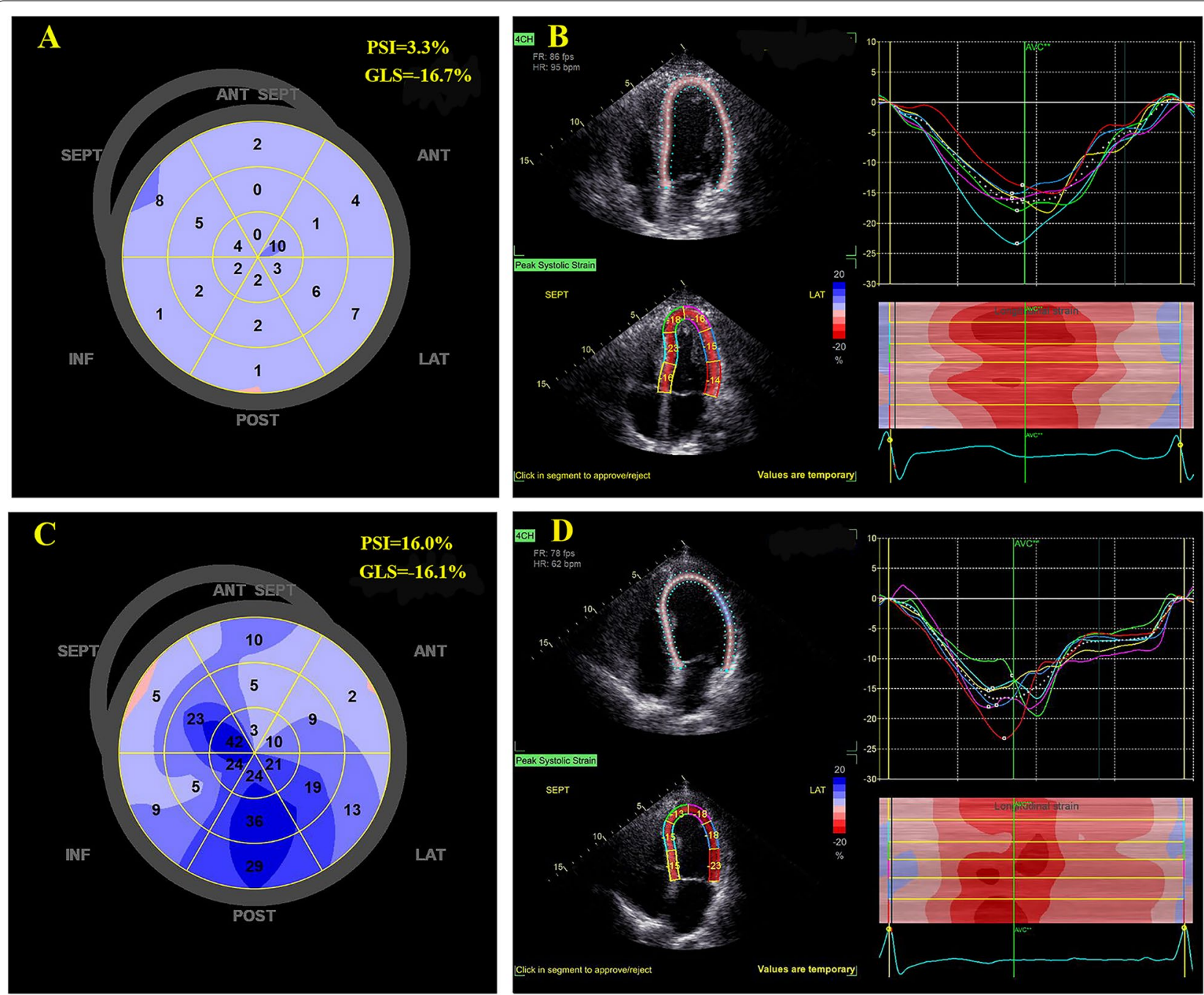

Fig. 1 Representative cases. The bull's eye map of post-systolic index (PSI) and longitudinal strain curves of six myocardial segments in the apical four-chamber view of the patients without $(\mathbf{A}, \mathbf{B})$ and with $(\mathbf{C}, \mathbf{D})$ major adverse cardiovascular events during follow-up. Both patients showed normal global longitudinal strain (GLS) and left ventricular ejection fraction (LVEF). A Normal bull's eye map of PSI with an average value of 3.3\%. B Normal strain curves with little or no sign of post-systolic shortening (PSS). C Abnormal bull's eye map of PSI with an increased average value of 16.0\%. D Abnormal strain curves where the green segment exhibits obvious PSS

groups. Categorical data are presented as percentages and statistically analyzed with the $\chi^{2}$ test or Fisher's exact test (as appropriate).

Receiver operator characteristic (ROC) curve analysis was performed to investigate the predictive ability of different indicators for adverse events. From the analysis of the ROC, areas under the curve (AUC) and $95 \%$ confidence intervals were obtained. By maximization of Youden's index, the optimal cutoff values with specificity or sensitivity were calculated. Comparison of AUC was performed using the method of DeLong in MedCalc. This cutoff value was used to construct Kaplan-Meier survival curves, and associations between the respective echocardiographic parameters and endpoints were compared by the log-rank test. Cox proportional hazards method was used to calculate hazard ratios (HRs) and 95\% confidence intervals (CIs). For multivariable COX proportional hazards regression analyses, LVEF and variables with significant $\mathrm{P}$ value $<0.05$ in univariable analyses were included in the multivariable models to detect independent risk factors for predicting adverse events. Restricted cubic spline model was constructed to analyze the relationship between PSI and the hazard ratio of adverse events. A two-way random-effects model was used with absolute agreement-type analysis for intraclass correlation 
coefficient (ICC). A two-tailed $p$ value $<0.05$ was considered a statistically significant difference.

\section{Results}

\section{Baseline patient characteristics}

A total of 297 participants diagnosed with stable CAD were examined by conventional echocardiography and STE. Of these participants, 78 were excluded because of exclusion criteria and 15 patients lost to follow-up. A total of 204 patients (mean age: $59 \pm 10$ years, $73 \%$ men) were finally evaluated in this study (Additional file 1: Fig. S2). During a median follow-up of 24 months (IQR 19-28 months), 30 patients (14.7\%) reached the composite endpoint, including 5 patients underwent heart failure, 13 patients experienced myocardial infarction, 4 patients admitted for stroke, and 8 patients died. The cause of death was cardiac in 4 patients, noncardiac in 1 patient and unknown in the remaining 3 patients.

Patient characteristics stratified by follow-up outcome are summarized in Table 1. Patients with events were generally older, showing a higher proportion of New York Heart Association (NYHA) functional class III-IV, a lower absolute GLS $--15.2 \pm 3.1 \%$ vs. $-17.4 \pm 2.8 \%$, $p<0.05)$, a higher PSI $(12.8 \%$ [IQR, $7.4-15.0 \%]$ vs. $6.3 \%$ [IQR, 4.4-9.3\%], $p<0.05)$ and more LV walls with PSS than patients without events. No significant differences were found regarding $\mathrm{E} / \mathrm{A}, \mathrm{E} / \mathrm{e}^{\prime}$, ventricular wall thickness, left ventricular volumes and LVEF between the two groups $(p>0.05)$.

In total, there are 99 (48.5\%) patients with no PSS, 62 (30.4\%) patients with $1-2$ walls of PSS and 43 (21.1\%) patients with $\geq 3$ walls of PSS. As shown in Table 2, patients with $\geq 3$ walls of PSS had a higher proportion

Table 1 Baseline characteristics stratified by follow-up outcome

\begin{tabular}{|c|c|c|c|c|}
\hline & $\begin{array}{l}\text { Overall population } \\
(n=204)\end{array}$ & With event $(n=30)$ & Without events ( $n=174)$ & $p$ value \\
\hline \multicolumn{5}{|l|}{ Baseline clinical } \\
\hline Age, year & $59 \pm 10$ & $64 \pm 10$ & $58 \pm 10$ & $0.009^{*}$ \\
\hline Male & $148(73)$ & $21(70)$ & $127(73)$ & 0.735 \\
\hline Body mass index, $\mathrm{kg} / \mathrm{m}^{2}$ & $22.9 \pm 2.7$ & $22.8 \pm 2.9$ & $23.0 \pm 2.6$ & 0.814 \\
\hline Systolic blood pressure, mm Hg & $129 \pm 17$ & $134 \pm 18$ & $128 \pm 17$ & 0.096 \\
\hline Diastolic blood pressure, $\mathrm{mm} \mathrm{Hg}$ & $78 \pm 11$ & $79 \pm 14$ & $77 \pm 11$ & 0.551 \\
\hline Heart rate, bpm & $70 \pm 10$ & $70 \pm 11$ & $71 \pm 11$ & 0.729 \\
\hline Smoke & $50(24.5)$ & $7(23.3)$ & $43(24.7)$ & 0.871 \\
\hline Hypertension & $119(58.3)$ & $22(73.3)$ & $97(55.7)$ & 0.071 \\
\hline Diabetes & $72(35.3)$ & $15(50.0)$ & $57(32.8)$ & 0.068 \\
\hline ACEI/AT2-antagonists & $81(39.7)$ & $16(53.3)$ & $65(37.4)$ & 0.099 \\
\hline Beta-blockers & $121(59.3)$ & $20(66.7)$ & $101(58.0)$ & 0.375 \\
\hline Calcium blocker & $99(48.5)$ & $12(40.0)$ & $87(50.0)$ & 0.311 \\
\hline Aspirin & $168(82.4)$ & $25(83.3)$ & $143(82.2)$ & 0.879 \\
\hline \multicolumn{5}{|l|}{ Revascularization therapy } \\
\hline $\mathrm{PCl}$ & $33(16.2)$ & $7(23.3)$ & $26(14.9)$ & 0.269 \\
\hline CABG & $90(44.1)$ & $15(50.0)$ & $75(43.1)$ & 0.482 \\
\hline Carotid plaque & $128(62.7)$ & $20(66.7)$ & $108(62.1)$ & 0.630 \\
\hline ST depression $\geq 0.5 \mathrm{~mm}$ & $77(37.7)$ & $13(43.3)$ & $64(36.8)$ & 0.494 \\
\hline T wave inversion $\geq 1 \mathrm{~mm}$ & $67(32.8)$ & $14(46.7)$ & $53(30.5)$ & 0.081 \\
\hline Pathological Q wave $\geq 0.04 \mathrm{~s}$ & $32(15.7)$ & $4(13.3)$ & $28(16.1)$ & 0.701 \\
\hline \multicolumn{5}{|l|}{ Location of significant stenosis } \\
\hline LAD & $152(74.5)$ & $26(86.7)$ & $126(72.4)$ & 0.098 \\
\hline LCX & $114(55.9)$ & $21(70.0)$ & $93(53.4)$ & 0.092 \\
\hline RCA & $111(54.4)$ & $21(70.0)$ & $90(51.7)$ & 0.063 \\
\hline NYHA functional class III-IV & $61(29.9)$ & $16(53.3)$ & $45(25.9)$ & $0.002^{*}$ \\
\hline
\end{tabular}

Data are presented as mean \pm SD or median (interquartile range) for continuous variables, and count (\%) for categorical variables

ACEl: angiotensin-converting enzyme inhibitors; AT: angiotensin; PCl: percutaneous coronary intervention; CABG: coronary artery bypass grafting; LAD: left anterior descending artery; LCX: left circumflex artery; RCA: right coronary artery; NYHA: New York Heart Association; IVS: interventricular septum; LVPW: left ventricular posterior ventricular wall; LVEDD: left ventricular end-diastolic diameter; LVEDV: left ventricular end-diastolic volume; LVESV: left ventricular end-systolic volume; LVMI: left ventricular mass index; LVEF: left ventricular ejection fraction; GLS: global longitudinal strain; PSI: post-systolic index; PSS: post-systolic shortening

P-value marks trend for events group vs. without events group $\left({ }^{*} p<0.05\right)$ 
Table 2 Baseline characteristics stratified by left ventricle walls with pathological PSS

\begin{tabular}{|c|c|c|c|c|}
\hline Walls with presence of PSS & No walls $(n=99)$ & $1-2$ walls $(n=62)$ & $\geq 3$ walls $(n=43)$ & $p$ value \\
\hline Age, year & $58 \pm 10$ & $59 \pm 10$ & $62 \pm 11$ & 0.222 \\
\hline Male & $72(73)$ & $44(71)$ & $32(74)$ & 0.925 \\
\hline Body mass index, $\mathrm{kg} / \mathrm{m}^{2}$ & $22.7 \pm 2.5$ & $23.3 \pm 3.0$ & $23.1 \pm 2.7$ & 0.353 \\
\hline Systolic blood pressure, mm Hg & $128 \pm 18$ & $129 \pm 17$ & $130 \pm 16$ & 0.795 \\
\hline Diastolic blood pressure, $\mathrm{mm} \mathrm{Hg}$ & $78 \pm 11$ & $77 \pm 10$ & $78 \pm 12$ & 0.749 \\
\hline Heart rate, bpm & $71 \pm 10$ & $70 \pm 9$ & $70 \pm 10$ & 0.797 \\
\hline Smoke & $29(29.3)$ & $10(16.1)$ & $11(25.6)$ & 0.165 \\
\hline Hypertension & $55(55.6)$ & $35(56.5)$ & $29(67.4)$ & 0.392 \\
\hline Diabetes & $36(36.4)$ & $16(25.8)$ & $20(46.5)$ & 0.088 \\
\hline Carotid plaque & $60(60.6)$ & $38(61.3)$ & $30(69.8)$ & 0.561 \\
\hline ST depression $\geq 0.5 \mathrm{~mm}$ & $38(39.4)$ & $21(32.3)$ & $18(41.9)$ & 0.697 \\
\hline T wave inversion $\geq 1 \mathrm{~mm}$ & $40(40.4)$ & $20(32.3)$ & $18(41.9)$ & 0.503 \\
\hline Pathological Q wave $\geq 0.04 \mathrm{~s}$ & $15(15.2)$ & $8(12.9)$ & $9(20.9)$ & 0.528 \\
\hline \multicolumn{5}{|l|}{ Location of significant stenosis } \\
\hline LAD & $70(71.7)$ & $47(75.8)$ & $35(81.4)$ & 0.390 \\
\hline LCX & $52(52.5)$ & $34(54.8)$ & $29(67.4)$ & 0.247 \\
\hline RCA & $48(48.5)$ & $35(56.5)$ & $28(65.1)$ & 0.174 \\
\hline NYHA functional class III-IV & $20(20.2)$ & $23(37.1)$ & $18(47.9)^{\#}$ & $0.012^{*}$ \\
\hline \multicolumn{5}{|l|}{ Echocardiography } \\
\hline IVS, mm & $10 \pm 1$ & $10 \pm 1$ & $11 \pm 1$ & 0.065 \\
\hline LVPW, mm & $10 \pm 3$ & $10 \pm 1$ & $10 \pm 1$ & 0.933 \\
\hline LVEDD, mm & $45 \pm 6$ & $45 \pm 5$ & $46 \pm 4$ & 0.385 \\
\hline LVEDV, mL & $88 \pm 22$ & $86 \pm 22$ & $95 \pm 27$ & 0.154 \\
\hline LVESV, mL & $35 \pm 11$ & $35 \pm 10$ & $41 \pm 13^{\# \&}$ & $0.008^{*}$ \\
\hline LVMI, $\mathrm{g} / \mathrm{m}^{2}$ & $87.6(75.6,100.0)$ & $89.9(77.0,109.7)$ & $95.9(79.2,118.1)$ & 0.060 \\
\hline$E / A$ & $0.8(0.7,0.9)$ & $0.8(0.6,1.0)$ & $0.7(0.6,0.9)^{\#}$ & $0.040^{*}$ \\
\hline
\end{tabular}

Data are presented as mean $\pm S D$ or median (interquartile range) for continuous variables, and count (\%) for categorical variables

LAD: left anterior descending artery; LCX: left circumflex artery; RCA: right coronary artery; NYHA: New York Heart Association; IVS: interventricular septum; LVPW: left posterior ventricular wall; LVEDD: left ventricular end-diastolic diameter; LVEDV: left ventricular end-diastolic volume; LVESV: left ventricular end-systolic volume; LVMI: left ventricular mass index; E/A: mitral inflow peak early velocity/mitral inflow peak late velocity; E/e': mitral inflow peak early velocity/mitral annular peak early velocity; LVEF: left ventricular ejection fraction; GLS: global longitudinal strain

$\# p<0.05$ versus no walls group. ${ }^{\&} p<0.05$ versus $1-2$ walls group

of NYHA functional class III-IV and a lower E/A ratio (0.7 [IQR, 0.6-0.9] vs. 0.8 [IQR, 0.7-0.9], $p<0.05)$ than patients with no PSS. Increasing numbers of LV walls with PSS were related to a higher incidence of adverse events, a lower LVEF, a higher LVESV and a lower GLS, such that participants with no PSS had a mean GLS of $-17.7 \pm 2.4 \%$, those with $1-2$ wall of PSS had a mean GLS of $-17.4 \pm 2.8 \%$ and those with $\geq 3$ walls of PSS had a mean GLS of $-15.2 \pm 3.5 \%$ ( $p$ trend $<0.05$ ). To some extent, these results indicated that patients with $\geq 3$ walls of PSS had impaired LV function compared with patients with $<3$ walls of PSS. No significant differences were found regarding left ventricular wall thickness, $E / \mathrm{e}^{\prime}$ across groups.
Event risk prediction by conventional echocardiographic and AFI parameters in stable CAD

ROC curves analyses of multiple parameters to predict adverse events in stable $\mathrm{CAD}$ are illustrated in Table 3. Among these echocardiography parameters, PSI $(\mathrm{AUC}=0.76$ [PSI] vs. 0.55 [LVEF], $p=0.003 ; 0.76$ [PSI] vs. $0.54[\mathrm{E} / \mathrm{A}], p=0.002 ; 0.76[\mathrm{PSI}]$ vs. $0.53\left[\mathrm{E} / \mathrm{e}^{\prime}\right]$, $p=0.002$ ) was superior to conventional parameters in predicting adverse events. The cutoff value was $10.4 \%$ for PSI with a sensitivity of $63.3 \%$ and specificity of 81.0\%. Similarly, GLS (AUC $=0.70$ [GLS] vs. 0.55 [LVEF], $p=0.040 ; 0.70$ [GLS] vs. 0.54 [E/A], $p=0.027 ; 0.70$ [GLS] vs. $\left.0.53\left[\mathrm{E} / \mathrm{e}^{\prime}\right], p=0.049\right)$ was also superior to conventional parameters in predicting adverse events. The cutoff 
Table 3 Receiver operating characteristic curve analysis of multiple parameters to predict adverse events

\begin{tabular}{lllllllll}
\hline & AUC & $\mathbf{9 5 \%} \mathbf{C l}$ & $\boldsymbol{p}$ value & $\boldsymbol{p}$ value (VS GLS) & $\boldsymbol{p}$ value (VS PSI) & Cutoff value & Sensitivity & Specificity \\
\hline LVEF, \% & 0.55 & $0.48-0.62$ & 0.334 & $0.040^{*}$ & $0.003^{*}$ & 60 & 70.0 & 46.6 \\
E/A & 0.54 & $0.47-0.61$ & 0.440 & $0.027^{*}$ & $0.002^{*}$ & 0.8 & 56.7 & 55.2 \\
E/e' & 0.53 & $0.46-0.60$ & 0.648 & $0.049^{*}$ & $0.002^{*}$ & 12.2 & 50.0 & 75.9 \\
GLS, $-\%$ & 0.70 & $0.63-0.76$ & $<0.001^{*}$ & $/$ & 0.249 & 15.4 & 56.7 & 77.0 \\
PSI, \% & 0.76 & $0.70-0.82$ & $<0.001^{*}$ & 0.249 & $/$ & 10.4 & 63.3 & 81.0
\end{tabular}

AUC: area under the curve; Cl: confidence interval; LVEF: left ventricular ejection fraction; E/A: mitral inflow peak early velocity/mitral inflow peak late velocity; E/e': mitral inflow peak early velocity/mitral annular peak early velocity; GLS: global longitudinal strain; PSI: post-systolic index

${ }^{*} p<0.05$

value was $-15.4 \%$ for GLS with a sensitivity of $56.7 \%$ and specificity of $77.0 \%$, whereas no significant difference was found between PSI and GLS (AUC $=0.76$ [PSI] vs. 0.70 [GLS], $p=0.249$ ) (Additional file 1: Fig. S3).

\section{Impact of conventional echocardiographic and AFI} parameters on the adverse events in stable CAD

Kaplan-Meier event-free survival curves are shown in Fig. 2. All spackle tracking indices (GLS, PSI and numbers of LV walls with PSS) had significant differences in survival time at different levels (log-rank $p<0.05$ for all parameters), whereas conventional parameters (LVEF, $\mathrm{E} / \mathrm{A}$ ) except $\mathrm{E} / \mathrm{e}^{\prime}$ showed no significant differences in survival time at different levels $(\log$-rank $p>0.05)$.

In univariable Cox regression analysis (Table 4), age (HR 1.06, 95\%CI 1.01-1.10, $p=0.008$ ), hypertension (HR 2.38, 95\%CI 1.01-5.59, $p=0.046$ ), NYHA functional class III-IV (HR 2.24, 95\%CI 1.05-4.76, $p=0.036$ ), GLS
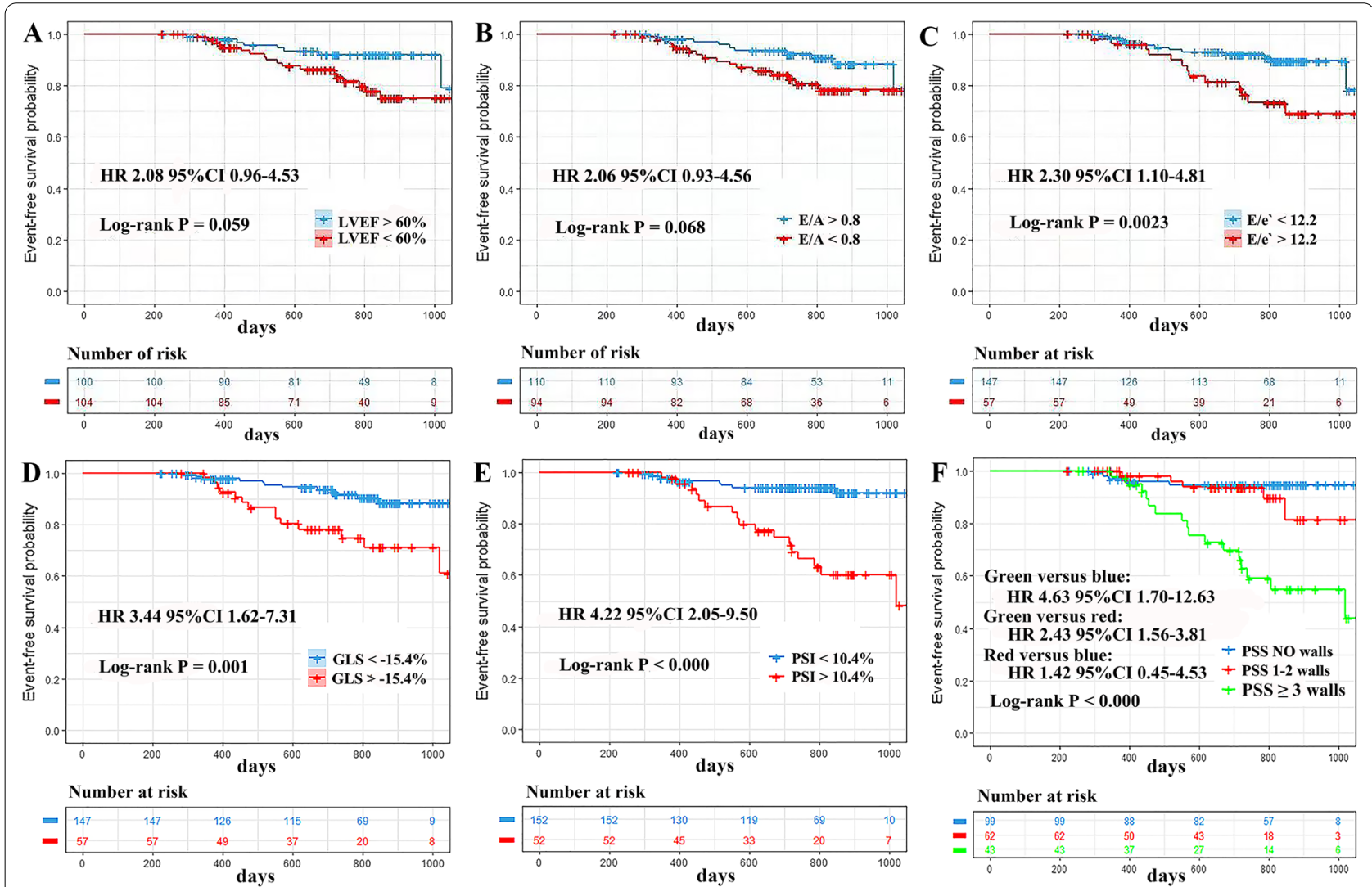

Fig. 2 Event-free survival. Kaplan-Meier survival curves for adverse events prediction by (A) LVEF; (B) E/A; (C) E/e'; (D) GLS; (E) PSI; (F) the number of left ventricle walls with PSS. LVEF: left ventricular ejection fraction; E/A: mitral inflow peak early velocity/mitral inflow peak late velocity; E/e': mitral inflow peak early velocity/mitral annular peak early velocity; GLS: global longitudinal strain; PSI: post-systolic index; PSS: post-systolic shortening 
Table 4 Cox regression analysis of multiple variables on the composite endpoint

\begin{tabular}{|c|c|c|c|c|c|c|}
\hline & \multicolumn{2}{|c|}{ Univariable analysis } & \multicolumn{4}{|c|}{ Multivariable analysis } \\
\hline & $\mathrm{HR}(95 \% \mathrm{Cl})$ & $P$ value & $\begin{array}{l}\text { Model } 1 \\
\text { HR (95\% Cl) }\end{array}$ & $P$ value & $\begin{array}{l}\text { Model } 2 \\
\text { HR (95\% Cl) }\end{array}$ & $P$ value \\
\hline Male & $1.06(0.46-2.40)$ & 0.898 & & & & \\
\hline Age & $1.06(1.01-1.10)$ & $0.008^{*}$ & $1.03(0.99-1.07)$ & 0.137 & $1.03(0.99-1.07)$ & 0.102 \\
\hline Body mass index, $\mathrm{kg} / \mathrm{m}^{2}$ & $1.00(0.87-1.14)$ & 0.952 & & & & \\
\hline Heart rate, bpm & $0.99(0.95-1.03)$ & 0.602 & & & & \\
\hline Systolic blood pressure, $\mathrm{mmHg}$ & $1.02(1.00-1.04)$ & 0.060 & & & & \\
\hline Diastolic blood pressure, $\mathrm{mmHg}$ & $1.03(1.00-1.06)$ & 0.081 & & & & \\
\hline Revascularization therapy & $1.42(0.59-3.41)$ & 0.429 & & & & \\
\hline Hypertension & $2.38(1.01-5.59)$ & $0.046^{*}$ & $2.12(0.86-5.25)$ & 0.102 & $1.71(0.69-4.28)$ & 0.250 \\
\hline Diabetes & $1.65(0.78-3.45)$ & 0.187 & & & & \\
\hline Carotid plaque & $1.22(0.55-2.68)$ & 0.625 & & & & \\
\hline Number of coronary stenoses & $1.18(0.84-1.66)$ & 0.336 & & & & \\
\hline NYHA functional class III-IV & $2.24(1.05-4.76)$ & $0.036^{*}$ & $1.45(0.66-3.18)$ & 0.350 & $1.29(0.59-2.80)$ & 0.523 \\
\hline LVEF, \% & $0.94(0.88-1.01)$ & 0.104 & $0.97(0.90-1.05)$ & 0.422 & $0.97(0.90-1.05)$ & 0.508 \\
\hline$E / A$ & $0.22(0.04-1.22)$ & 0.082 & & & & \\
\hline$E / e^{\prime}$ & $0.97(0.87-1.07)$ & 0.560 & & & & \\
\hline GLS, - \% & $0.85(0.76-0.95)$ & $0.004^{*}$ & $0.95(0.84-1.08)$ & 0.419 & $0.96(0.85-1.09)$ & 0.965 \\
\hline PSI, \% & $1.19(1.09-1.29)$ & $0.001^{*}$ & $1.15(1.04-1.27)$ & $0.005^{*}$ & & \\
\hline Per 1 increase in number of walls with PSS & $1.69(1.39-2.05)$ & $<0.000^{*}$ & & & $1.52(1.21-1.91)$ & $<0.000^{*}$ \\
\hline
\end{tabular}

HR: hazard ratio; Cl: confidence interval; NYHA: New York Heart Association; LVEF: left ventricular ejection fraction; E/A: mitral inflow peak early velocity/mitral inflow peak late velocity; E/e': mitral inflow peak early velocity/mitral annular peak early velocity; GLS: global longitudinal strain; PSI: post-systolic index; PSS: post-systolic shortening

${ }^{*} p<0.05$

(HR 0.85, 95\%CI 0.76-0.95, $p=0.004$ ), PSI (HR 1.19, $95 \%$ CI $1.09-1.29, p=0.001)$ were significantly associated with adverse events, while no significant associations were found in LVEF, E/A and E/e' ( $p>0.05$ for all). Per 1 increase in number of LV walls with PSS, the risk of adverse events increased by 1.69 times (95\% CI 1.39-2.05, $p<0.000)$. LVEF and all variables with $p<0.05$ in univariable Cox regression analysis were included in multivariable Cox regression analysis. Both the number of walls with PSS (per 1 increase in the number of walls with PSS: HR 1.52, 95\%CI 1.21-1.91, $p<0.000$ ) and PSI (HR $1.15,95 \%$ CI 1.04-1.27, $p=0.005$ ) remained independent predictors, but no remained significant prediction was observed in GLS after adjustment.

PSI showed a non-Gaussian distribution, which was successfully converted to a Gaussian distribution using logarithmic transformation. The association between PSI and risk of adverse events was evaluated on a continuous scale with restricted cubic spline curves based on Cox proportional hazards models. The increasing PSI was associated with significantly increased risk of adverse events. Figure 3 shows a significant $p<0.001$ for nonlinearity PSI-HR.

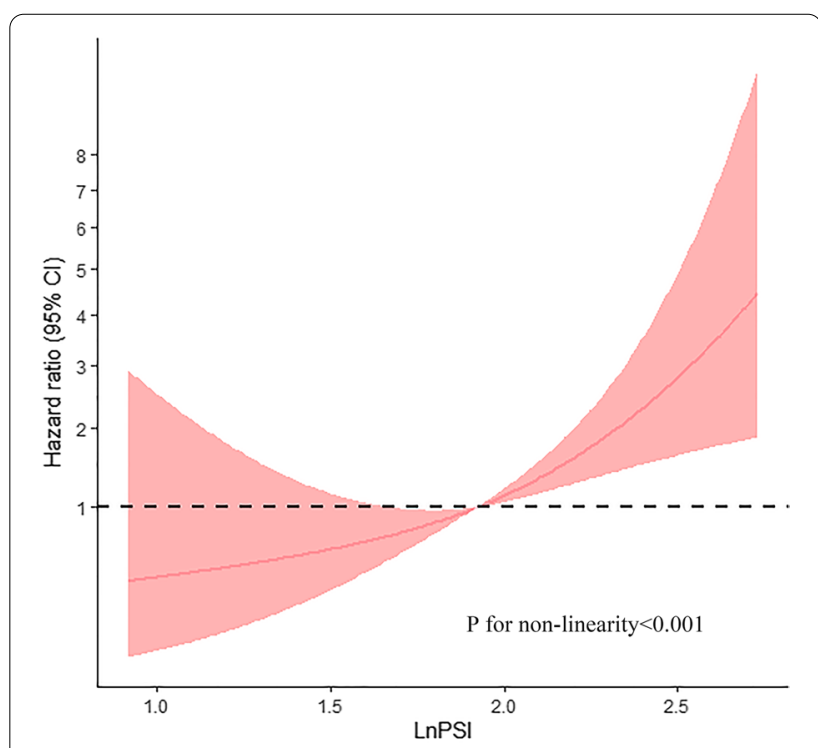

Fig. 3 Restricted cubic splines of nonlinear association between LnPSI and hazard ratio. The bold red lines indicate the pooled restricted cubic spline model, and the red filled area indicates the 95\% confidence intervals of the pooled curve. PSI: post-systolic index 
The prediction performance of PSS was further explored in patients with the absolute value of GLS $>15.4 \%$, representing patients with sub-clinical myocardial dysfunction. In these patients, a significant prognostic superiority was found in PSI compared with GLS $(\mathrm{AUC}=0.73[\mathrm{PSI}]$ vs. $0.58[\mathrm{GLS}], p=0.024)$. These results indicated that for patients with normal or mildly reduced GLS, the prognostic value of PSS may be superior to GLS. Furthermore, Kaplan-Meier survival curves showed that patients with both GLS and PSI beyond the prognostic cutoff values had significantly higher adverse events risk than patients with only GLS beyond the prognostic cutoff value (HR 4.41, 95\%CI 1.42-13.72, Log-rank $p=0.005$ ). For patients with the absolute value of GLS $>15.4 \%, \geq 3$ walls of PSS showed a higher risk of adverse events than 1-2 walls of PSS (HR 3.95, 95\%CI 0.94-16.61) and no walls of PSS (HR 2.32, 95\%CI 1.20-4.49) (log-rank $p=0.010$ ) (Fig. 4).

\section{Inter-observer and intraobserver variability analyses}

As demonstrated in Additional file 1: Table S1, both intra- and inter-observer intraclass correlation coefficients indicate good or excellent reliability for all strain parameters.

\section{Discussion}

This prospective study explored the prognostic value of myocardial deformation parameters in stable CAD patients with preserved systolic function. The primary findings are illustrated as follows, (1) the novel strain parameters, including GLS, PSS and PSI obtained from the AFI algorithm can early identify myocardial dysfunction and predict adverse events in stable CAD with normal LVEF; (2) PSI and the number of LV walls with PSS are independent predictors of the endpoint; (3) the cutoff of PSI in predicting the adverse events is $10.4 \%$ with sensitivity $63.3 \%$ and specificity $81.0 \%$; (4) for patients with normal or mildly reduced GLS, the prognostic value of PSS may be superior to GLS.

Coronary artery disease (CAD) is a pathological process characterized by atherosclerotic plaque accumulation in the epicardial arteries. The disease can have long, stable periods but can also become unstable at any time, leading to major adverse cardiovascular events such as myocardial infarction (MI), stroke and death [23]. Therefore, event risk assessment is an important step during the work-up for the patients with stable CAD since it has a major impact on the subsequent therapeutic decisions.

STE is recommended for the early diagnosis and risk stratification in a variety of cardiovascular diseases in recent years [5, 24]. Compared to the traditional echocardiographic parameters, it could provide more sensitive parameters in early identifying subtle myocardial damage, particularly contributing to improve the prognostic assessment in the patients with preserved $\operatorname{LVEF}[25,26]$. In the present study, speckle tracking AFI was applied in the routine work-up for patients with stable CAD to provide multiple deformation parameters fast and accurately.
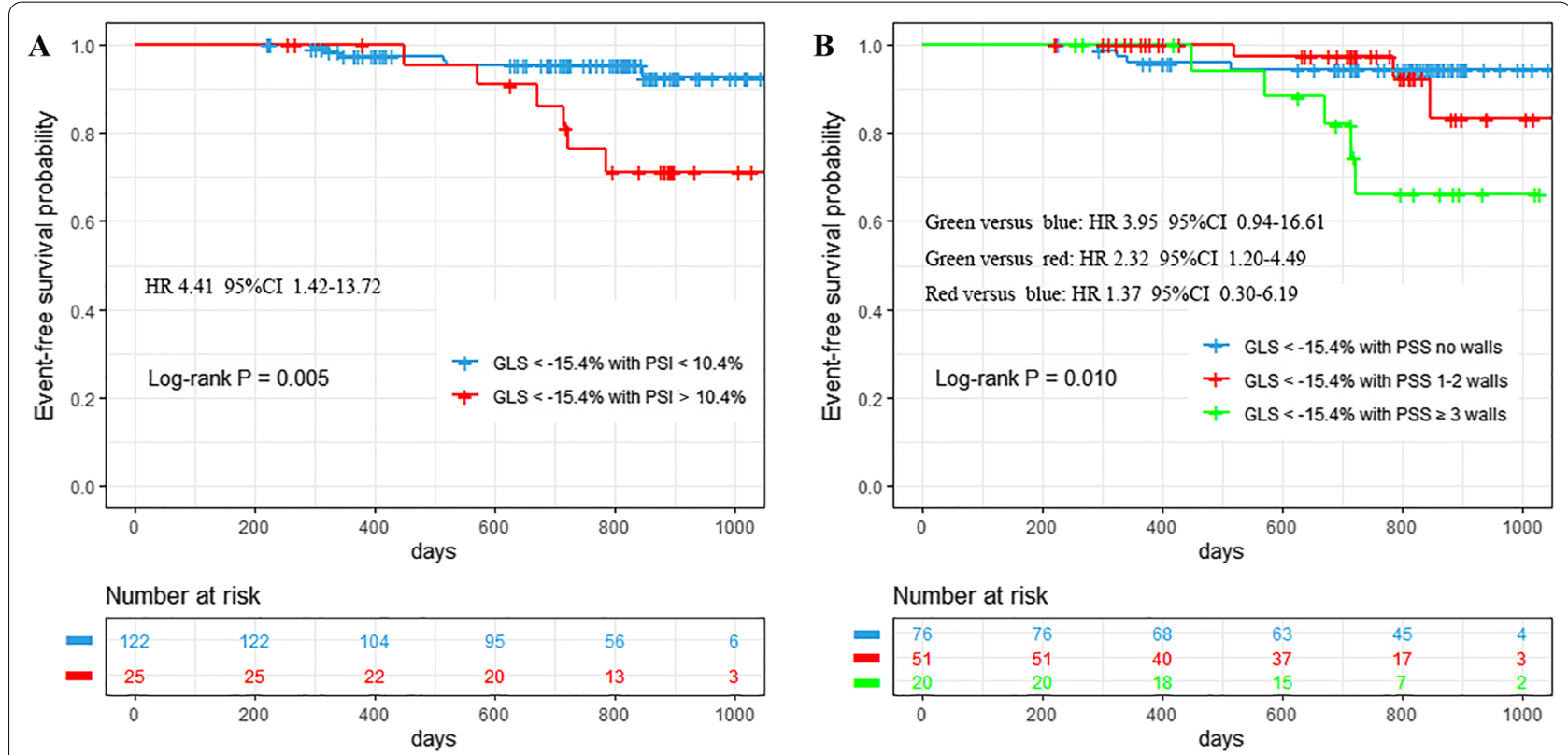

Fig. 4 Event-free survival. Kaplan-Meier survival curves for adverse events prediction in patients with stratification of GLS>15.4\% by (A) GLS; (B) the number of left ventricle walls with PSS. GLS: global longitudinal strain; PSI: post-systolic index; PSS: post-systolic shortening 
Consistent with previous studies, our study confirmed that both GLS and PSS obtained from the AFI algorithm could predict adverse events in stable CAD patients with normal LVEF. We found that increasing numbers of LV walls with PSS were related to impaired LV function. Differently, the predictive power of AFI parameters was further compared in the present study. Our results strongly supported PSS and PSI as independent predictors rather than GLS, indicating PSS may be more sensitive to pathological alterations and potentially ischemia than GLS [7]. In fact, the superiority of PSS to systolic longitudinal strain has also been indicated in other clinical scenarios. Voigt et al. [27] evaluated strain parameters during dobutamine stress in 44 patients with known and suspected coronary artery disease and compared the diagnostic accuracies of the deformation parameters with that of perfusion scintigraphy. PSS was observed during stress in all ischemic segments, and PSS parameters such as the PSI were better for identifying stress-induced ischemia than maximal strain in systole. It has recently been found that PSS may persist after brief ischemia even when peak systolic strain is recovered [14, 28]. For this reason, PSS is expected to be a valuable indicator and recognize the myocardial ischemic insults accurately. Brainin et al. [29] explored the diagnostic and prognostic potential of PSS in 293 patients with suspected stable angina pectoris. During a median follow-up of 3.5 years, a total of 25 patients $(8.5 \%)$ experienced major adverse cardiovascular events. Although the presence of PSS remains an independent predictor of adverse events when GLS was added to the adjusted model, no significant difference in the prognostic performance for the primary end point was found between GLS and PSS. By contrast, the superiority of PSI to GLS in predicting adverse events was found in patients diagnosed with stable CAD with the absolute value of $\mathrm{GLS}>15.4 \%$ in the present study $(\mathrm{AUC}=0.73$ [PSI] vs. 0.58 [GLS], $p=0.024)$. In Cox regression analysis, both PSS and PSI are independent predictors rather than GLS. It is reported that absolute GLS $<12 \%$ represents severe systolic dysfunction and adverse prognosis, and $<15 \%-16 \%$ seems to represent risk in patients with relatively preserved EF [30,31]. Specifically, our results suggested that PSS may be a sensitive indicator to pathological alterations when strain in systole was preserved. PSS appears to provide prognostic information when GLS is normal or near normal. Our conclusion could be supported by another prospective study [32], where PSS provided prognostic information in general population with strain within normal range (GLS, $-17.1 \% \pm 2.9 \%$ ).

PSS was initially considered as a delayed but active contraction induced by ischemia [33]. However, many studies suggested that the contraction of the ischemic myocardium is affected not only by inherent contractility but also by tension from the surrounding non-ischemic myocardium [10, 34]. In a mathematical model study by Claus et al. [9], PSS was explained as passive recoil caused by the interaction between the ischemic and surrounding myocardium. Although the exact mechanism of whether PSS is caused by active contraction or passive recoil remains unclear, several studies demonstrated the close association of PSS and the adverse outcomes in type 2 diabetes [35], acute coronary syndrome [36] and even in the general population [32]. In our study, patients with pathological PSS had a lower systolic function. More importantly, we found that patients with an increasing number of walls with PSS had lower GLS and more adverse events. The incidence of events increased incrementally with the increase of PSI. These findings may involve two implications. First, PSS characterizes dysfunctional myocardial segments due to ischemic burden in the myocardium, which often occurs in conjunction with the relative reduction of longitudinal myocardial strain during ejection $[11,13]$. Second, it is reported that the extent of PSS detected by STE is consistent with the ischemic area and the damage degree [7]. Therefore, a high PSI or more pathological PSS involved chamber walls is directly related to the extent of impaired myocardial segments, indicating the worse outcome.

\section{Limitations}

There are still some limitations in the present study. First, the current study provides prognostic information on speckle tracking AFI multiparameter in patients with stable CAD. However, we believe it cannot be widely used for risk stratification in real-world clinical practice because of the strict inclusion and exclusion criteria in our study. Despite this, our findings confirmed the prognostic value of PSS in stable CAD patients with preserved systolic function, contributing to the risk stratification in those with relatively low-risk population. Also due to sample size limitations, the study used a combined end point including death and cardiovascular events, which may mask the predictive power for different endpoint events. Second, there may be overfitting in our multivariate model due to a lower incidence of adverse events. However, this could be acceptable when controlling for confounders as opposed to building prediction models [37]. Third, some studies suggested to identify pathologic from physiological PSS by analyzing the dynamic process of PSS. However, PSS is regarded as present if PSI is $>20 \%$ in the present study according to the criteria proposed by Voigt et al. We did not expire the relationship between the dynamic process of PSS and the adverse events. Last, it has recently been reported that early systolic lengthening (ESL) is also useful to detect myocardial ischemia and 
may provide some information on prognosis. Integrated analysis of myocardial deformational patterns should be explored in future studies.

\section{Conclusion}

Speckle tracking AFI could provide useful prognostic information in stable CAD patients with preserved LVEF by early identifying myocardial dysfunction with strain parameters. There is strong evidence of the prognostic value of PSS for predicting major adverse cardiovascular events. Increased PSI or a greater number of LV walls with PSS indicate an increased risk of adverse events, and the prognostic value of PSS may be superior to GLS in patients with normal or mildly reduced GLS.

\section{Abbreviations}

AFI: Automated functional imaging; AUC: Areas under the curve; CAD: Coronary artery disease; $\mathrm{Cl}$ : Confidence interval; E/A: Mitral inflow peak early velocity/mitral inflow peak late velocity; E/e': Mitral inflow peak early velocity/ mitral annular peak early velocity; GLS: Global longitudinal strain; HR: Hazard ratio; ICC: Intraclass correlation coefficient; IQR: Interquartile range; IVS: Interventricular septum; LAD: Left anterior descending artery; LCX: Left circumflex artery; LM: Left main artery; LV: Left ventricle; LVEDD: LV end-diastolic dimension; LVEDV: LV end-diastolic volumes; LVEF: Left ventricular ejection fraction; LVESV: LV end-systolic volumes; LVMI: LV mass index; LVPW: LV posterior wall; MACEs: Major adverse cardiovascular events; MI: Myocardial infarction; NYHA: New York Heart Association; PSI: Post-systolic index; PSS: Post-systolic shortening; RCA: Right coronary artery; ROC: Receiver operator characteristic curve; ROI: Region of interest; STE: Speckle tracking echocardiography; WMSI: Wall motion score index.

\section{Supplementary Information}

The online version contains supplementary material available at https://doi. org/10.1186/s13244-022-01174-y.

Additional file 1. Table S1. Inter- and intraobserver variability for automated functional imaging parameters. Fig. S1. Schematic drawing of measurement of post-systolic index (PSI). PSI was calculated as follows: [(peak negative strain in cardiac cycle-peak negative strain in systole)/ (peak negative strain in the cardiac cycle) $\times$ 100. Fig. S2. Flowchart of study population. CAD: coronary artery disease; LVEF: left ventricular ejection fraction. Fig. S3. Receiver operating characteristic curves of multiple parameters for evaluating adverse events. AUC: area under the curve; E/A: mitral inflow peak early velocity/mitral inflow peak late velocity; E/e': mitral inflow peak early velocity/mitral annular peak early velocity; LVEF: left ventricular ejection fraction; PSI: post-systolic index; GLS: global longitudinal strain.

\section{Authors' contributions}

$\mathrm{SL}, \mathrm{XH}, J Z, Y Z, W Z, Y L$ and $Y D$ helped in study conception and design, acquisition of data, analysis and interpretation of data and drafting of manuscript. $Y L$ and YD critically revised the manuscript. All authors read and approved the final manuscript.

\section{Funding}

Dr. Liu is supported by Natural Science Foundation of Hubei Province in China (grant number 2021 CFB336, 2021).

\section{Availability of data and materials}

The dataset used or analyzed during the current study are available from the corresponding author on reasonable request.

\section{Declarations}

Ethics approval and consent to participate

The Tongji Hospital Ethics Committee approved the study, and informed consent was obtained from all patients before the study.

\section{Consent for publication}

Informed consent for the procedures was signed from all patients or their families, and it was approved by the Institutional Review Board of The Tongji Hospital.

\section{Competing interests}

The authors declare that they have no competing interests.

Received: 17 November 2021 Accepted: 3 February 2022

Published online: 02 March 2022

\section{References}

1. Heart Disease and Stroke Statistics-2016 update a report from the American Heart Association. Circulation 2021.

2. Fox KAA, Metra M, Morais J, Atar D (2020) The myth of "stable" coronary artery disease. Nat Rev Cardiol 17:9-21

3. Clayton TC, Lubsen J, Pocock SJ et al (2005) Risk score for predicting death, myocardial infarction and stroke in patients with stable angina, based on a large randomised trial cohort of patients. BMJ 331:869

4. Task Force M, Montalescot G, Sechtem U et al (2013) 2013 ESC guidelines on the management of stable coronary artery disease: the Task Force on the management of stable coronary artery disease of the European Society of Cardiology. Eur Heart J 34:2949-3003

5. Singh A, Voss WB, Lentz RW, Thomas JD, Akhter N (2019) The diagnostic and prognostic value of echocardiographic strain. JAMA Cardiol 4:580-588

6. Antoni ML, Mollema SA, Delgado V et al (2010) Prognostic importance of strain and strain rate after acute myocardial infarction. Eur Heart J 31:1640-1647

7. Asanuma T, Nakatani S (2015) Myocardial ischaemia and post-systolic shortening. Heart 101:509-516

8. Rumbinaite E, Karuzas A, Verikas D et al (2020) Detection of functionally significant coronary artery disease: role of regional post systolic shortening. J Cardiovasc Echogr 30:131-139

9. Claus P, Weidemann F, Dommke C et al (2007) Mechanisms of postsystolic thickening in ischemic myocardium: mathematical modelling and comparison with experimental ischemic substrates. Ultrasound Med Biol 33:1963-1970

10. Voigt J (2003) Incidence and characteristics of segmental postsystolic longitudinal shortening in normal, acutely ischemic, and scarred myocardium. J Am Soc Echocardiogr 16:415-423

11. Pastore MC, Mandoli GE, Contorni F et al (2021) Speckle tracking echocardiography: early predictor of diagnosis and prognosis in coronary artery disease. Biomed Res Int 2021:6685378

12. Malagoli A, Fanti D, Albini A, Rossi A, Ribichini FL, Benfari G (2020) Echocardiographic strain imaging in coronary artery disease: the added value of a quantitative approach. Cardiol Clin 38:517-526

13. Asanuma T, Fukuta Y, Masuda K, Hioki A, Iwasaki M, Nakatani S (2012) Assessment of myocardial ischemic memory using speckle tracking echocardiography. JACC Cardiovasc Imaging 5:1-11

14. Kozuma A, Asanuma T, Masuda K, Adachi H, Minami S, Nakatani S (2019) Assessment of myocardial ischemic memory using three-dimensional speckle-tracking echocardiography: a novel integrated analysis of early systolic lengthening and postsystolic shortening. J Am Soc Echocardiogr 32:1477-1486

15. Chilingaryan AL, Tunyan LG, Shamoyan RV (2020) Role of segmental and global left ventricular strain in diagnosis of acute coronary syndrome. Kardiologiia 60:20-24

16. Feigenbaum H, Mastouri R, Sawada S (2012) A practical approach to using strain echocardiography to evaluate the left ventricle. Circ J 76:1550-1555 
17. Recommendations for cardiac chamber quantification by echocardiography in adults: an update from the American Society of Echocardiography and the European Association of, Cardiovascular Imaging. Eur Heart J Cardiovasc Imaging 17:412.

18. Weng Y, Liu Y, Deng Y, Lu S, Zhu Y (2021) Rapidly and accurately detecting significant coronary artery stenosis in patients with suspected stable coronary artery disease and normal segmental wall motion by speckle tracking automated functional imaging. Ultrasound Med Biol 47:546-555

19. Zhang J, Liu Y, Deng Y, Zhu Y, Sun R, Lu S (2021) Non-invasive global and regional myocardial work predicts high-risk stable coronary artery disease patients with normal segmental wall motion and left ventricular function. Front Cardiovasc Med 8:71154

20. Barbier P, Mirea O, Cefalu C, Maltagliati A, Savioli G, Guglielmo M (2015) Reliability and feasibility of longitudinal AFI global and segmental strain compared with 2D left ventricular volumes and ejection fraction: intraand inter-operator, test-retest, and inter-cycle reproducibility. Eur Heart J Cardiovasc Imaging 16:642-652

21. Skaarup KG, Iversen A, Jorgensen PG et al (2018) Association between layer-specific global longitudinal strain and adverse outcomes following acute coronary syndrome. Eur Heart J Cardiovasc Imaging 19:1334-1342

22. Levine GN, Bates ER, Blankenship JC et al (2011) 2011 ACCF/AHA/ SCAI Guideline for Percutaneous Coronary Intervention: a report of the American College of Cardiology Foundation/American Heart Association Task Force on Practice Guidelines and the Society for Cardiovascular Angiography and Interventions. Circulation 124:e574-651

23. Knuuti J, Wijns W, Saraste A et al (2020) 2019 ESC Guidelines for the diagnosis and management of chronic coronary syndromes. Eur Heart J 41:407-477

24. Pastore MC, De Carli G, Mandoli GE et al (2020) The prognostic role of speckle tracking echocardiography in clinical practice: evidence and reference values from the literature. Heart Fail Rev. https://doi.org/10.1007/ s10741-020-09945-9

25. Kalam K, Otahal P, Marwick TH (2014) Prognostic implications of global LV dysfunction: a systematic review and meta-analysis of global longitudinal strain and ejection fraction. Heart 100:1673-1680

26. Holzknecht M, Reindl M, Tiller C et al (2021) Global longitudinal strain improves risk assessment after ST-segment elevation myocardial infarction: a comparative prognostic evaluation of left ventricular functional parameters. Clin Res Cardiol. https://doi.org/10.1016/S0735-1097(21) 02669-3

27. Voigt JU, Exner B, Schmiedehausen K et al (2003) Strain-rate imaging during dobutamine stress echocardiography provides objective evidence of inducible ischemia. Circulation 107:2120-2126

28. Asanuma T, Uranishi A, Masuda K, Ishikura F, Beppu S, Nakatani S (2009) Assessment of myocardial ischemic memory using persistence of post-systolic thickening after recovery from ischemia. JACC Cardiovasc Imaging 2:1253-1261

29. Brainin P, Hoffmann S, Fritz-Hansen T, Olsen FJ, Jensen JS, BieringSorensen T (2018) Usefulness of postsystolic shortening to diagnose coronary artery disease and predict future cardiovascular events in stable angina pectoris. J Am Soc Echocardiogr 31:870-879.e873

30. Haugaa KH, Grenne BL, Eek CH et al (2013) Strain echocardiography improves risk prediction of ventricular arrhythmias after myocardial infarction. JACC Cardiovasc Imaging 6:841-850

31. Ersboll M, Valeur N, Mogensen UM et al (2013) Prediction of all-cause mortality and heart failure admissions from global left ventricular longitudinal strain in patients with acute myocardial infarction and preserved left ventricular ejection fraction. J Am Coll Cardiol 61:2365-2373

32. Brainin P, Biering-Sorensen SR, Mogelvang R, Sogaard P, Jensen JS, Biering-Sorensen T (2018) Postsystolic shortening by speckle tracking echocardiography is an independent predictor of cardiovascular events and mortality in the general population. J Am Heart Assoc. https://doi. org/10.1161/JAHA.117.008367

33. Skulstad $\mathrm{H}$, Edvardsen $\mathrm{T}$, Urheim $\mathrm{S}$ et al (2002) Postsystolic shortening in ischemic myocardium: active contraction or passive recoil? Circulation 106:718-724

34. Brainin $P$, Holm AE, Sengelov $M$ et al (2021) The prognostic value of myocardial deformational patterns on all-cause mortality is modified by ischemic cardiomyopathy in patients with heart failure. Int J Cardiovasc Imaging. https://doi.org/10.1007/s10554-021-02291-3
35. Brainin P, Jensen MT, Biering-Sorensen T et al (2020) Post-systolic shortening by speckle tracking echocardiography predicts cardiac events in type 2 diabetes. JACC Cardiovasc Imaging 13:1289-1291

36. Brainin P, Skaarup KG, Iversen AZ et al (2019) Post-systolic shortening predicts heart failure following acute coronary syndrome. Int J Cardiol 276:191-197

37. Vittinghoff E, McCulloch CE (2007) Relaxing the rule of ten events per variable in logistic and Cox regression. Am J Epidemiol 165:710-718

\section{Publisher's Note}

Springer Nature remains neutral with regard to jurisdictional claims in published maps and institutional affiliations.

\section{Submit your manuscript to a SpringerOpen ${ }^{\circ}$ journal and benefit from:}

- Convenient online submission

- Rigorous peer review

Open access: articles freely available online

- High visibility within the field

- Retaining the copyright to your article

Submit your next manuscript at $\boldsymbol{\nabla}$ springeropen.com 